\title{
International collaboration during the COVID-19 crisis: autumn 2020 developments
}

\author{
X. Cai ${ }^{1}$ (D) C. V. Fry ${ }^{2}$ (D) C. S. Wagner ${ }^{3}$
}

Received: 13 November 2020 / Accepted: 15 January 2021 / Published online: 14 February 2021 (c) Akadémiai Kiadó, Budapest, Hungary 2021

\begin{abstract}
After the initial shock of the early months of the global COVID-19 pandemic, international collaboration in COVID-19 research continues to show aberrant patterns compared to coronavirus research in pre-COVID times. The most affected nations tend to produce the greatest number of coronavirus articles, with output closely coupled to the rate of infection. COVID-19 research has fewer nations and smaller teams than pre-COVID research, a trend which intensifies throughout the pandemic. The United States remains the single largest contributor to the global publication output, but contrary to China's dominance in the initial months of the pandemic, China's contribution falls as the national COVID-19 caseload drops. China-USA collaborations drop as the pandemic continues, perhaps due to China's reduced rate of publication on the topic, and perhaps due to political obstacles, or a combination of these factors.
\end{abstract}

Keywords COVID-19 $\cdot$ International collaboration $\cdot$ Scientific research $\cdot$ Cooperation

\section{Introduction}

The COVID-19 pandemic has resulted in a 'firehose' of articles and data on the topic (Kupferschmidt 2020), propelled by shortened time-to-acceptance compared to pre-COVID-19 (Aviv-Reuven and Rosenfeld, 2020) and the growth of preprint platform usage (Fry and MacGarvie 2020). Zhang et al. (2020a) noted that historical patterns show that researchers have always responded quickly to public health emergencies with a sharp increase in the number of publications on the emergency topic, a finding we can confirm in the COVID19 era. In updating our earlier work (Fry et al. 2020), we find that the number of coronavirus publications has seen a great boom in 2020, rising at a spectacular rate from a total of 4,875 articles produced on the topic (preprint and peer reviewed) between January and mid-April to an overall sum of 44,013 by mid-July, and 87,515 by the start of October

C. S. Wagner

wagner.911@osu.edu

1 School of Public Affairs, Zhejiang University, Hangzhou 310058, China

2 Shidler College of Business, University of Hawai'i at Manoa, Honolulu, USA

3 John Glenn College of Public Affairs, Ohio State University, Columbus, OH 43210, USA 
2020 (in comparison, nanoscale science was a rapidly growing field in the 1990s, but it took more than 19 years to go from 4000 to 90,000 articles (Grieneisen and Zhang, 2011)). Given that the crisis is ongoing, that the world continues to require solutions, and that a travel ban remains in place, we expect to find that the trend towards smaller, more elite teams has continued, and that patterns of international collaborative partnerships mirror the trends in the rise and fall of COVID-19 cases.

Fry et al. (2020) showed that early COVID-19 research featured significant levels of China-USA collaboration, and that shortly into the initial weeks of the pandemic, researchers from the UK joined China-USA international teams. Liu et al. (2020) showed growth in what they call 'parachuting collaborations'- new connections not seen prior to the pandemic - which have dramatically increased during the pandemic. Together with the findings in Fry et al. (2020), this suggests that search and team formation patterns across the network changed to adapt to the needs of COVID-19 research, a finding also reported by Lee and Haupt (2020). Liu et al. (2020) further found that COVID-19 research papers were less likely to involve international collaboration than non-COVID-19 papers during the same time period, a finding reported by Aviv-Reuven and Rosenfeld (2020) as well, both supporting Fry et al. (2020) in the assertion that the urgency of the pandemic necessitates smaller, familiar teams who can work quickly to task.

Importantly, among the articles reviewing the publication patterns of COVID-19 work, all find differences among countries in the extent to which they produce coronavirus articles, as well as differences in participation rates among nations in international collaborations, as compared to before the pandemic. The trends diverge from pre-COVID-19 work in four main ways. First, Fry et al. (2020) showed that very few developing countries were involved in early COVID-19 research. Zhang et al. (2020b) confirmed Fry et al. in finding that the USA, China, and the UK are the three countries with the largest number of articles as well as the most international collaborations. Lee and Haupt (2020) found that many nations did not join the COVID-19 collaborations: those nations more affected by the crisis tended to participate more in international collaboration than their less affected counterparts. Second, a departure from pre-COVID-19 research is found in the smaller teams publishing together, a finding confirmed by Aviv-Reuven and Rosenfeld (2020). This is likely due to the need for rapidity in responding to the crisis. Third, a departure is seen in smaller and more centralized networks, where leadership reverts to elite institutions in leading nations. Fourth, COVID-19 research exhibits higher novelty than coronavirus research before the pandemic (Liu et al. 2020), especially for China (Zhang et al. 2020a, b). In this paper we continue this line of inquiry and explore how trends in coronavirus research continue to develop through the pandemic. While previous research has focused on the earliest months of the pandemic, we explore whether the trends observed in these early months have continued through the pandemic period, or returned to pre-pandemic dynamics.

As the pandemic continued to ravage nations like the USA, India, and some European countries throughout 2020, the very rapid growth in the number of coronavirus-related publications slowed for preprints. ${ }^{1}$ Following the decline in the global share of COVID-19 cases and the rise in articles from a larger set of countries, articles from China represented a smaller share of world articles than in the early months of the pandemic. In contrast, the USA and European nations turned out increasing numbers of COVID-19 articles, a finding particularly true of Italy which rapidly increased its output during COVID-19.

\footnotetext{
1 The drop may also be due to lags in peer review processes. The authors could not distinguish between these possible mechanisms.
} 
Table 1 Production of coronavirus articles by time period

\begin{tabular}{|c|c|c|c|c|c|}
\hline \multicolumn{6}{|c|}{ Number of publications with author affiliation details } \\
\hline \multirow[t]{2}{*}{ Source } & \multirow{2}{*}{$\begin{array}{l}\text { Pre-COVID-19 (January } \\
1 \text { 2018-December } 31 \\
\text { 2019) }\end{array}$} & \multicolumn{4}{|c|}{ COVID-19 } \\
\hline & & Total & Period 1 & Period 2 & Period 3 \\
\hline Published items & 6,980 & 74,771 & 2,728 & 32,420 & 39,623 \\
\hline Published items (formal) & 5,292 & 57,833 & 1,508 & 22,817 & 33,508 \\
\hline $\begin{array}{l}\text { Preprints (arXiv / bioRxiv / } \\
\text { medRxiv / ChemRxiv) }\end{array}$ & & 12,744 & 2,147 & 6,718 & 3,879 \\
\hline Combined & 6,980 & 87,515 & 4,875 & 39,138 & 43,502 \\
\hline
\end{tabular}

COVID-19 is separated into 3 Periods: Period I, from January 1 to April 8; Period II, from April 9 to July 12; and Period III, from July 13 to October 5. Periods 1-3 for preprints are almost the same as published items except for the separating date between Period I and Period II is April 25. Formal publications are defined as articles, journal articles, and research support (in PMC) that are published in journals

Consistent with our previous findings in Fry et al. (2020) but somewhat surprisingly, team size continued to shrink, with the number of authors, number of nations, and the rate of international-teamed articles continuing to decrease from pre-COVID-19. In line with the drop in Chinese-authored articles, and in contrast to the volume of USA-China collaboration in the early months of the pandemic, the rate at which these two countries collaborate slowed as the pandemic continued through 2020.

\section{Methodology}

Coronavirus-related documents from Elsevier Scopus, Clarivate Web of Science, PubMed Central (PMC), as well as (arXiv, medRxiv, ChemRxiv, BioRxiv) preprints accessed from Dimensions were collected using the same search strategy as Fry et al. (2020). Data were collected for multiple time periods, enabling us to analyze research publications during COVID-19 by periods. We define four periods in the data corresponding to (1) preCOVID-19 as articles produced between 2018 and 2019; (2) Period I as January 1-April 8, 2020; (3) Period II as April 9-July 12, 2020; and (4) Period III as July 13-October 5, 2020. Author affiliation data is used to ascertain the nations producing articles with a full count going to a participating nation. These same data were used to assess international collaboration. To illustrate the changing contributions and collaboration patterns of different nations, we focus much of our analysis on China, the first economy that suffered from the pandemic, as well as the 10 most-affected countries (the United States (USA), India, France, Brazil, the United Kingdom (UK), Italy, Spain, Russia, Belgium, and Poland) in terms of cumulative COVID-19 cases to date as recorded by Johns Hopkins Coronavirus Resource Center (https://coronavirus.jhu.edu/data/new-cases). We also generate variables on publication team size, or the number of authors per article. We do this by counting the number of names in the author list of each publication in the dataset.

Table 1 shows the numbers of articles by time period. The table reveals that researchers are continuing to produce a large number of articles in COVID-19 related topics in up to 10 months after the start of the pandemic, but at a slower rate of growth than seen in Spring 2020. 
Table 2 National contributions to formal publications

\begin{tabular}{|c|c|c|c|c|c|}
\hline & \multicolumn{5}{|c|}{ Number of publications (percentage of all publications) } \\
\hline & \multirow[t]{2}{*}{ Pre-COVID-19 } & \multicolumn{4}{|c|}{ COVID-19 (up until 5 October 2020) } \\
\hline & & Total & Period I & Period II & Period III \\
\hline Overall & 5175 & 52,697 & 1362 & 20,337 & 30,998 \\
\hline China & $1240(24 \%)$ & $9223(17.5 \%)$ & $645(47.4 \%)$ & $3597(17.7 \%)$ & $4981(16.1 \%)$ \\
\hline USA & $1741(33.6 \%)$ & $17,129(32.5 \%)$ & $316(23.2 \%)$ & $6580(32.4 \%)$ & $10,233(33 \%)$ \\
\hline Europe & $1364(26.4 \%)$ & $17,687(33.6 \%)$ & $274(20.1 \%)$ & $6917(34 \%)$ & $10,496(33.9 \%)$ \\
\hline UK & $262(5.1 \%)$ & $3504(6.6 \%)$ & $67(4.9 \%)$ & $1465(7.2 \%)$ & $1972(6.4 \%)$ \\
\hline Italy & $172(3.3 \%)$ & $5636(10.7 \%)$ & $61(4.5 \%)$ & $2196(10.8 \%)$ & $3379(10.9 \%)$ \\
\hline India & $189(3.7 \%)$ & $3511(6.7 \%)$ & $39(2.9 \%)$ & $1366(6.7 \%)$ & $2106(6.8 \%)$ \\
\hline France & $220(4.3 \%)$ & $2813(5.3 \%)$ & $46(3.4 \%)$ & $1155(5.7 \%)$ & $1612(5.2 \%)$ \\
\hline Brazil & $127(2.5 \%)$ & $1375(2.6 \%)$ & $18(1.3 \%)$ & $479(2.4 \%)$ & $878(2.8 \%)$ \\
\hline Spain & $146(2.8 \%)$ & $2278(4.3 \%)$ & $23(1.7 \%)$ & $872(4.3 \%)$ & $1383(4.5 \%)$ \\
\hline Russia & $51(1 \%)$ & $273(0.5 \%)$ & $9(0.7 \%)$ & $103(0.5 \%)$ & $161(0.5 \%)$ \\
\hline Poland & $50(1 \%)$ & $497(0.9 \%)$ & $4(0.3 \%)$ & $204(1 \%)$ & $289(0.9 \%)$ \\
\hline Belgium & $78(1.5 \%)$ & $862(1.6 \%)$ & $17(1.2 \%)$ & $355(1.7 \%)$ & $490(1.6 \%)$ \\
\hline 11 Countries Total & $3608(69.7 \%)$ & $39,536(75 \%)$ & $1069(78.5 \%)$ & $15,359(75.5 \%)$ & $23,108(74.5 \%)$ \\
\hline
\end{tabular}

We include only formal articles that report affiliations with country information. COVID-19 is separated into 3 Periods: Period I, from January 1 to April 8; Period II, from April 9 to July 12; and Period III, from July 13 to October 5

In earlier work, Fry et al. (2020) included preprints as part of the assessment of national contributions. In this article, when calculating the national contributions (using a full counting method) and international collaboration, we exclude preprints. This choice reflects the fact that about $50 \%$ of preprints do not provide information on author affiliations, which could lead to a distorted count. Moreover, the data on formal publications is truncated at the time of writing, and so we have no way of knowing how many preprints do not end up in peer-reviewed journals. In addition, many have noted that the quality of the preprints is questionable. For these reasons, we have dropped preprints from the calculation and focus on formal publication records in the following analysis.

\section{Results}

\section{National contributions}

Table 2 shows the contributions of major nations and regions to coronavirus research, based on formal publications. A more intuitive view in Fig. 1 depicts the relative share of each nation/region. After the sharp increase in COVID-19 publication production of China shortly after the outbreak of COVID-19, the share of Chinese articles decreased in the later months of 2020 , from $47.4 \%$ of all articles in first three months to $16.1 \%$ in July-October 2020. In contrast, countries such as India, Brazil, France, Spain, Poland, and Belgium gained greater visibility in later periods of the pandemic. In particular, Italy greatly 


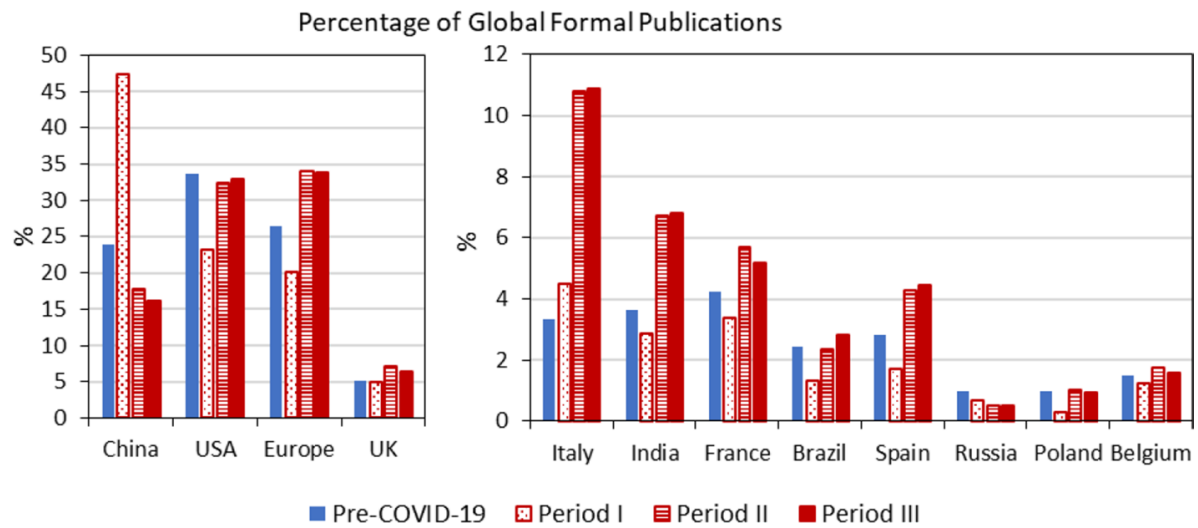

Fig. 1 Percentage of global formal publications of a given country/region. The number of publications of a country is full counted. The data is separated into 4 periods: Pre-COVID-19 (January 1, 2018-December 31, 2019), Period I (January 1-April 8), Period II (April 9-July 12), and Period III (July 13-October 5)

increased the volume of publications through the months of the pandemic. Interestingly, Russia exhibits the opposite trend. Although home to a large number of COVID-19 cases, Russian researchers were contributing a lower share of coronavirus publications than in pre-COVID-19.

In general, and somewhat surprisingly, the trend of publication shares of each country (Fig. 1) mirrors the trend of newly confirmed cases of COVID-19 in the selected countries. For example, there were very few newly confirmed cases of COVID-19 in China since Period II, in line with the drop in production of new articles. In contrast, India and Brazil reported rapid growth in the number of COVID-19 cases in Period II and III, and also showed a great boom in global shares of publications since Period II. European countries, including the UK, Italy, France, and Spain, which suffered from first and second waves of the pandemic in April and September respectively, also showed significant increase in contributions to the global COVID-19 research base in these time periods.

\section{Team size and international collaboration}

Studies have shown that scientific team sizes have grown over time (Wuchty et al. 2007), and that international collaboration has grown over time (Wagner et al. 2015), but this pattern appears to be reversed in coronavirus research during the COVID-19 pandemic. Table 3 and Fig. 2 show the result of an analysis of team size where we see that both author and country-level team sizes continue to shrink for those engaged in coronavirus-related research. The rate of international teaming shown in all articles remains much lower during COVID-19 than in the pre-COVID-19 period. Interestingly, as shown in Fig. 2d, e, the average number of countries involved in a given article actually increases throughout the pandemic - articles are more internationally linked-while the number of authors decreases throughout the pandemic after Period II, to far below pre-pandemic levels. In fact, in articles with an international team of coauthors, the number of participating countries continues to rise to slightly above the pre-pandemic levels by Autumn 2020. This implies that teams have consolidated, but a broader set of nations participates in team formation over time due to the need for diverse expertise and data. 
Table 3 Team size in formal publications

\begin{tabular}{|c|c|c|c|c|}
\hline & \multicolumn{4}{|c|}{ Mean (standard deviations) } \\
\hline & \multirow[t]{2}{*}{ Pre-COVID-19 } & \multicolumn{3}{|l|}{ COVID-19 } \\
\hline & & Period I & Period II & Period III \\
\hline \multicolumn{5}{|l|}{ Overall } \\
\hline Number of authors & $\begin{array}{c}7.55 \\
(5.67)\end{array}$ & $\begin{array}{c}7.28 * \\
(7.79)\end{array}$ & $\begin{array}{l}6.09 * * * \\
(6.55)\end{array}$ & $\begin{array}{l}6.27 * * * \\
(6.6)\end{array}$ \\
\hline Number of countries & $\begin{array}{c}1.49 \\
(0.99)\end{array}$ & $\begin{array}{l}1.41 * * * \\
(0.91)\end{array}$ & $\begin{array}{l}1.46^{*} \\
(1.3)\end{array}$ & $\begin{array}{l}1.45^{* *} \\
(1.25)\end{array}$ \\
\hline $\begin{array}{l}\text { International team }(=1 \text { if article has } \\
\text { an international team })\end{array}$ & $\begin{array}{c}0.31 \\
(0.46)\end{array}$ & $\begin{array}{l}0.26 * * * \\
(0.44)\end{array}$ & $\begin{array}{l}0.245^{* * *} \\
(0.43)\end{array}$ & $\begin{array}{l}0.25 * * * \\
(0.43)\end{array}$ \\
\hline \multicolumn{5}{|c|}{ Just sample of articles with international teams } \\
\hline Number of authors & $\begin{array}{c}9.61 \\
(9.24)\end{array}$ & $\begin{array}{l}9.43 \\
(8.5)\end{array}$ & $\begin{array}{l}8.26^{* * * *} \\
(8.02)\end{array}$ & $\begin{array}{l}8.13 * * * \\
(7.94)\end{array}$ \\
\hline Number of countries & $\begin{array}{c}2.54 \\
(1.23)\end{array}$ & $\begin{array}{c}2.54 \\
(1.18)\end{array}$ & $\begin{array}{l}2.86^{* * * *} \\
(2.06)\end{array}$ & $\begin{array}{l}2.8 * * * \\
(1.94)\end{array}$ \\
\hline
\end{tabular}

$*$, **, *** denote that the difference between means in a two-sided $T$-test of each COVID-19 period versus Pre-COVID-19 is significant at $90 \%, 95 \%, 99 \%$ confidence level

Figure 3 shows in more detail the percentage of international teamed articles as a share of all coronavirus articles before and during the COVID-19 period for the most affected countries and regions. China has the lowest percentage share of international articles, although the rate of participation increases slightly during the pandemic. Belgium, Russia, and the UK have the highest percentage share of international collaborations compared to national output. The data reveals that, throughout the pandemic, the USA, Italy, France, Brazil, Spain, Russia, and Belgium decline in their rate of international collaboration, perhaps because the travel ban made it difficult for people to travel to a collaborative site. Poland increases its international collaboration throughout the pandemic.

As the most productive countries in the world in terms of scientific publications, China and the USA have come to be active collaborators across a number of topics, and this rise in their collaborative relationship has attracted a great deal of attention (see Cao et al. 2020). Fry et al. (2020) also reported more intensive collaboration between China and the USA on coronavirus research during the early COVID-19 period as compared to just before the pandemic. Table 4 reveals the changing rates of China-USA collaboration through the pandemic. As mentioned, the early months of the COVID-19 period saw an intensification of the China-USA collaborative relationship. However, this leveled off in the later months of the pandemic, returning to levels lower than the pre-pandemic levels of collaboration between the two countries. While this drop in the rate of collaboration between the two nations mirrors the drop in publications produced by Chinese researchers, it may also reflect political factors and limitations to travel between the two countries in particular. 


\section{All Formal Published Articles}

(a)

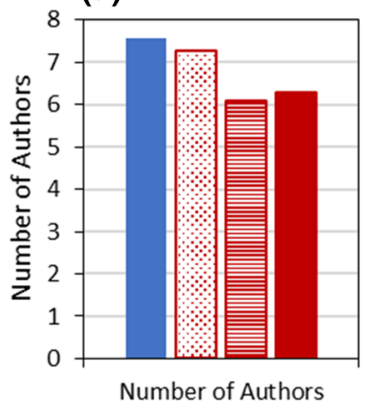

(b)

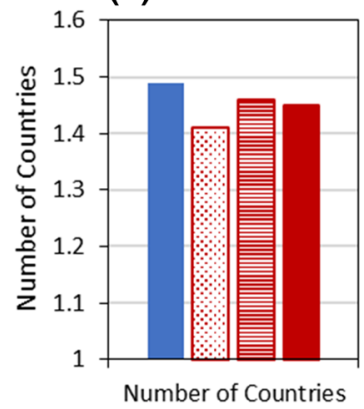

(c)

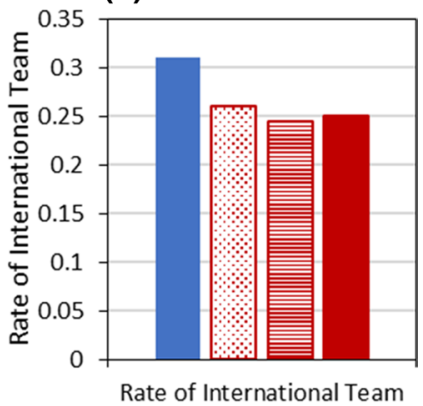

Formal Published Articles with International Teams

(d)

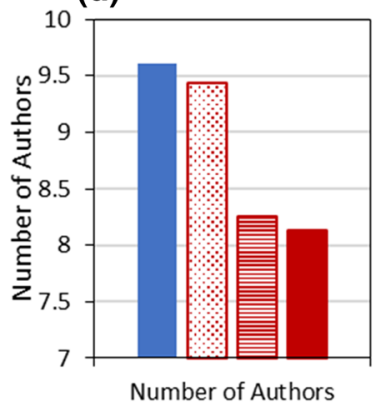

(e)

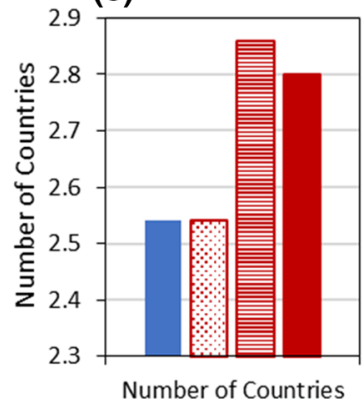

- pre-COVID-19

口COVID-19(I)

曰COVID-19(II)

口COVID-19 (III)

Fig. 2 Team size in all formal publications and in formal publications with international teams. a, b show the average number of authors and countries of all formal publications; $\mathbf{c}$ shows the rate of internationalteamed articles in all formal publications; $\mathbf{d}$, e show the average number of authors and countries in international-teamed formal publications

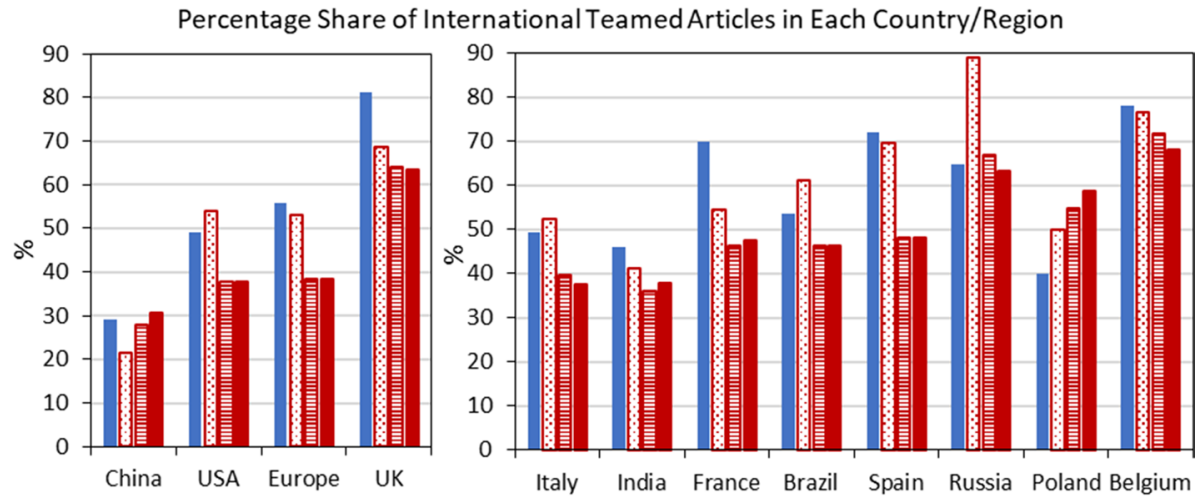

\section{口 Pre-COVID-19 OPeriod I 目Period II Deriod III}

Fig. 3 Percentage share of international teamed articles in each country/region based on formal publications 
Table 4 Ratio of China-USA collaboration in formal coronavirus related publications

\begin{tabular}{|c|c|c|c|c|}
\hline & \multicolumn{4}{|c|}{ Mean (standard deviations) } \\
\hline & \multirow[t]{2}{*}{ Pre-COVID-19 } & \multicolumn{3}{|l|}{ COVID-19 } \\
\hline & & Period I & Period II & Period III \\
\hline \multirow[t]{2}{*}{ Rate in overall articles } & 0.038 & $0.048^{* *}$ & $0.027^{* * *}$ & $0.025^{* * *}$ \\
\hline & $(0.19)$ & $(0.215)$ & $(0.161)$ & $(0.157)$ \\
\hline \multirow[t]{2}{*}{ Rate in international teamed articles } & 0.2 & $0.184^{* * *}$ & 0.109 & $0.101^{* *}$ \\
\hline & $(0.325)$ & $(0.388)$ & $(0.311)$ & $(0.301)$ \\
\hline \multirow[t]{2}{*}{ Rate in Chinese articles } & 0.537 & 0.478 & 0.539 & 0.515 \\
\hline & $(0.499)$ & $(0.501)$ & $(0.499)$ & $(0.5)$ \\
\hline Rate in USA articles & 0.228 & $0.388^{* * *}$ & 0.219 & $0.204^{*}$ \\
\hline
\end{tabular}

* $, * *, * * *$ denote that the difference between mean rates of each COVID-19 period versus pre-COVDI-19 is significant at $90 \%, 95 \%, 99 \%$ confidence level

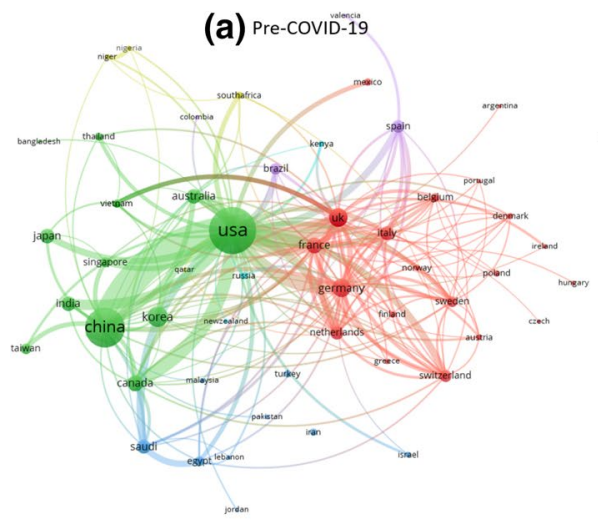

(C) COVID-19 Period Htpous

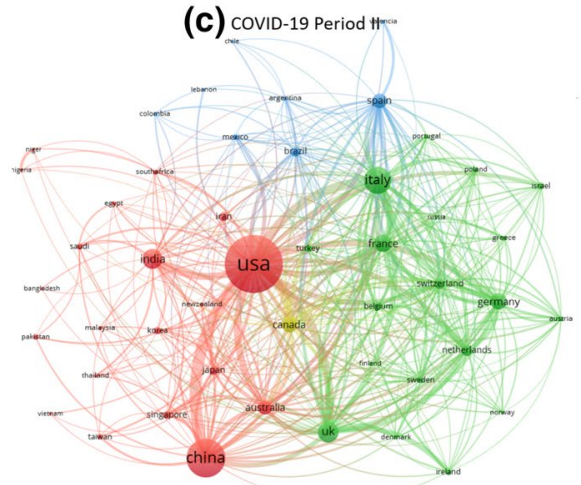

(b) covID-19 Period I
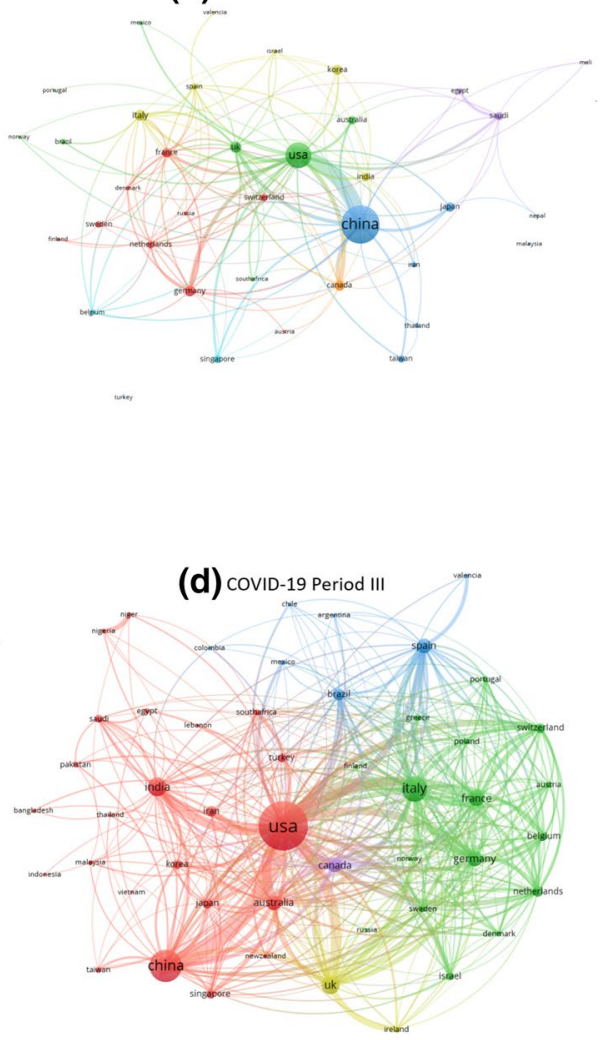

Fig. 4 International Collaborative Networks at Country Level based on Formal Published Articles. Networks in a Pre-COVID-19, b COVID-19 Period I, c COVID-19 Period II, and d COVID-19 Period III are constructed based on all publications of countries with at least $0.3 \%$ of publications in that period, i.e., 15 , 5, 70, 100 articles. Node sizes are proportional to number of publications of the countries. Links between nodes show the strength of collaboration between two countries. Only links with values higher than 5,2 , 10,15 are shown 


\section{International collaborative networks}

Figure 4 shows the networks of nations cooperating on coronavirus (based on formal publications). The network dynamics change rapidly from pre-COVID-19 (Fig. 4a) to COVID19 Period I (Fig. 4b), when the network is sparse and dominated by China. The change shown between Fig. 4a and Fig. 4b reflects the shock of the initial pandemic where we see a sharp decrease in ties between countries within the network just after the outbreak of COVID-19. This drop reflects the initial lock-down period as well as the difficulty of gathering data in the first days. However, we do see that China-USA collaborations are resilient in the earliest months. As the COVID-19 pandemic spread, more nations join or rejoin the global collaborative network in coronavirus research. Two nations, India and France, grow their role in the network compared to pre-COVID-19. As the network evolves, despite China's lower publication shares and increasing shares of European countries, China-USA collaboration remains the most prominent link in the network. However, researchers in the USA grow their links with other nations over time, diversifying the network and mitigating the centrality of the China-USA relationship. Network 4d in COVID-19 Period III looks very similar in structure and density to the pre-COVID network in $4 \mathrm{a}$, suggesting that the world research enterprise had revived to pre-COVID structure by Autumn 2020.

\section{Discussion and conclusion}

COVID-19 research teams and international collaboration show a quixotic pattern of rapid change in reaction to the pandemic. In the first months of the COVID-19 pandemic, the majority of articles and international collaboration was limited to the largest, most affected nations (China and the USA) and those with strong research capacity (the UK). As the pandemic has dragged on around the world throughout 2020, we see the numbers of articles rise but more slowly than in the earliest months. That said, over time the research system recovers from the initial shock and many more nations begin to collaborate and publish results. By Autumn 2020, the network is similar in density to that of coronavirus research communities in the pre-COVID period.

We document three features of coronavirus research that merit attention. First, consistent with prior research on epidemics such as Ebola and COVID-19 (Fry 2019; Fry and MacGarvie 2020), we document that the number of articles produced by nations is closely correlated to the number of infections in that nation. We attribute this to two possibilities: one is the need for data, and the second is that governments are funding research in response to the rate of infection, but these interpretations require more research.

Second, we document that COVID-19 team sizes drop and continue to drop through the pandemic. This is unexpected. If we were to see the system returned to its former state, we would see team sizes growing. We attribute this to the need for speedy results as the pandemic infections grew rapidly. Larger teams have higher transaction costs associated with communication, data sharing, testing, and writing; smaller teams can mitigate the drag of communication costs. These changes also may be due to physical and political obstacles to collaboration.

Third, the rate of international collaboration amongst articles continues to drop throughout the pandemic. This could be due to physical or political obstacles. The physical obstacles have included travel bans across the world, hindering researcher mobility. Generally, we know that the vast majority of research partnerships begin face-to-face (Wagner 2009), 
thus the travel bans may be significantly curtailing formation of research collaborations. There may also be significant political influence on the patterns. Political obstacles to collaboration may be more subtle than the physical ones, but they may also be influencing the rate of activity and connection. In April 2020 for example, the Chinese government required that all COVID-19 articles be centrally reviewed, perhaps slowing the rate of collaboration between countries (although we did not study this question). Political statements in the United States questioning aspects of the China-USA scientific relationship may also be hurting the initiation of new partnerships as Lee and Haupt (2020) noted; this despite researchers' calls for intensifying the relationship (Evans et al. 2020). Increased scrutiny of China-USA collaborations by the USA government have worried some researchers (Silver 2020). The rate of rapid funding of research may be closely tied to infection rates as a political response to the emergency. Future research will explore these possibilities as explanations for changes in the patterns of collaboration.

\section{References}

Aviv-Reuven, S., \& Rosenfeld, A. (2020). Publication patterns' changes due to the COVID-19 pandemic: A longitudinal and short-term scientometric analysis. arXiv preprint arXiv:2010.02594.

Cao, C., Baas, J., Wagner, C. S., \& Jonkers, K. (2020). Returning scientists and the emergence of China's science system. Science and Public Policy, 47(2), 172-183.

Grieneisen, M. L., \& Zhang, M. (2011). Nanoscience and nanotechnology: Evolving definitions and growing footprint on the scientific landscape. Small (Weinheim an der Bergstrasse, Germany), 7(20), 2836-2839.

Evans, T. S., Shi, Z., Boots, M., Liu, W., Olival, K. J., Xiao, X., et al. (2020). Synergistic China-US ecological research is essential for global emerging infectious disease preparedness. EcoHealth, 1-14.

Fry, C. V. (2019). Crisis. Collaboration and the Very Invisible College: Evidence from Ebola, Preprint.

Fry, C. V., Cai, X., Zhang, Y., \& Wagner, C. S. (2020). Consolidation in a crisis: Patterns of international collaboration in early COVID-19 research. PLOS ONE, 15(7), e0236307.

Fry, C.V., \& MacGarvie, M. (2020). Drinking from the Firehose: Preprints, Chinese researchers, and the diffusion of knowledge in COVID-19. Preprint.

Kupferschmidt, K. (2020). Preprints being a 'firehose' of outbreak data. Science, 367(6481), 963-964.

Lee, J. J., \& Haupt, J. P. (2020). Scientific globalism during a global crisis: research collaboration and open access publications on COVID-19. High Education. https://doi.org/10.1007/s10734-020-00589-0.

Liu, M., Bu, Y., Chen, C., Xu, J., Li, D., Leng, Y., et al. (2020). Can pandemics transform scientific novelty? Evidence from COVID-19. arXiv preprint. arXiv:2009.12500.

Silver, A. (2020). US crackdown harms chinese collaborations. Nature, 583, 341-342. https://doi. org/10.1038/d41586-020-02015-y.

Wagner, C. S., \& Fukuyama, F. (2009). The new invisible college: Science for development. Washington: Brookings Institution Press.

Wagner, C. S., Park, H. W., \& Leydesdorff, L. (2015). The continuing growth of global cooperation networks in research: A conundrum for national governments. PLOS ONE, 10(7), e0131816. https://doi. org/10.1371/journal.pone.0131816.

Wuchty, S., Jones, B. F., \& Uzzi, B. (2007). The increasing dominance of teams in production of knowledge. Science, 316(5827), 1036-1039.

Zhang, L., Zhao, W., Sun, B., Huang, Y., \& Glänzel, W. (2020a). How scientific research reacts to international public health emergencies: A global analysis of response patterns. Scientometrics. https://doi. org/10.1007/s11192-020-03531-4.

Zhang, Y., Cai, X., Fry, C. V., Wu M. J., Wagner, C. S. (preprint 2020b). Topic evolution, disruption and resilience in early COVID-19 Research. SSRN. Retrieved from https://papers.ssrn.com/sol3/paper s.cfm?abstract_id=3675020. 